\title{
The Computerized Tomographic Assessment of Brain Infarcts
}

\author{
D.E. TUBMAN, R. ETHIER, D. MELANÇON, G. BÈLANGER and S. TAYLOR
}

SUMMARY: The computed tomographic (CT) examinations of one hundred and sixty patients with a clinical diagnosis of cerebral infarction were reviewed. $A$ characteristic $C T$ pattern was noted. Infused scans and follow-up studies allowed for considerable accuracy in diagnosis.

RESUME: Nous présentons une revue des résultats de lomodensitométrie sur ordinateur de cent-soixante patients dont le diagnostic est celui de thrombose cérébrale. Nous notons un pattern caractéristique à la tomodensitométrie. Les études après infusions, et le suivi, permettent une exactitude considérable dans le diagnostic.

\footnotetext{
From the Departments of Radiology, Health Sciences Centre. Winnipeg. Manitoba, and Neuroradiology. Montreal Neurological Hospital, Montreal, Quebec.

Reprint requests to Dr. David E. Tubman, Department of Radiology. Health Sciences Centre, 700 William Avenue, Winnipeg, Manitoba, Canada R3E 0Z3.
}

\section{INTRODUCTION}

Ischemic cerebral infarcts often have a characteristic appearance on computed tomography (CT) due to a combination of features: distribution, configuration, density, mass effect, and enhancement following contrast infusion. Computed tomography allows for accurate diagnosis of infarcts and the possible evaluation of their natural history.

\section{MATERIALS AND METHODS}

One hundred and sixty in-patients of the Montreal Neurological Hospital with a clinical diagnosis of cerebral infarction were included in this retrospective study. This number represented approximately $40 \%$ of the total cases of infarcts assessed. The remainder were out-patients and patients from other institutions and were excluded because of uncertain diagnoses and/or incomplete histories. A total of two hundred and sixty-eight examinations were obtained on either the EMI Mark I or 5005 CT Scanners. Infused studies were performed following intravenous injection of Hypaque - $\mathrm{R} 60 \%$ given as a bolus of $1.9 \mathrm{ml}$ per kilogram of body weight immediately before the examination followed by an intravenous drip of contrast media during the scan. One hundred and seventy-five scans were done as initial examinations with ninety-three followup studies.

\section{RESULTS}

Eighty-eight males and seventy-two females ranging in age from 18 to 86 years with a mean age of 57 years were examined.

A total of thirty carotid angiograms were performed. Three were normal. Ten were abnormal and specific for cerebral infarction (Taveras \& Wood, 1976). Seventeen were non-specific, showing either ipsilateral carotid occlusion or mass effect.

Forty-four radionuclide scintigraphic static and flow scans were performed using technetium 99 pertechnetate. All scans were performed within the first four weeks following onset of symptoms. Six scans were normal. Fifteen scans showed localized areas of increased or decreased uptake of isotope in a pattern consistent with infarction. Twenty-three scans showed decreased flow to the appropriate cerebral hemisphere.

The CT scans were grouped according to the interval between onset of the disease and time of scan (Table 1).

TABLE 1

Time Between Onset of Disease and Scan

\begin{tabular}{|c|c|c|}
\hline & \multicolumn{2}{|c|}{ Number of Scans } \\
\hline & Plain & Infused \\
\hline Less than 24 hours & 27 & 8 \\
\hline I day to 1 week & 42 & 11 \\
\hline 1 to 2 weeks & 22 & 15 \\
\hline 2 to 3 weeks & 17 & 12 \\
\hline 3 to 4 weeks & 11 & 8 \\
\hline 4 weeks to 3 months & 20 & 8 \\
\hline 3 to 6 months & 13 & 3 \\
\hline Greater than 6 months & 39 & 14 \\
\hline
\end{tabular}

Twenty scans were obtained for infarcts in the brain stem. All were normal.

Most acute infarcts (less than six weeks) presented as areas of low density (Hounsfield number (HU) 10 to 30 units) in the geographic distribution of a major cerebral blood vessel, often extending to the periphery.

One case presented with a central area of high density and surrounding lucency. This was thought to represent a hemorrhage into an acute ischemic 


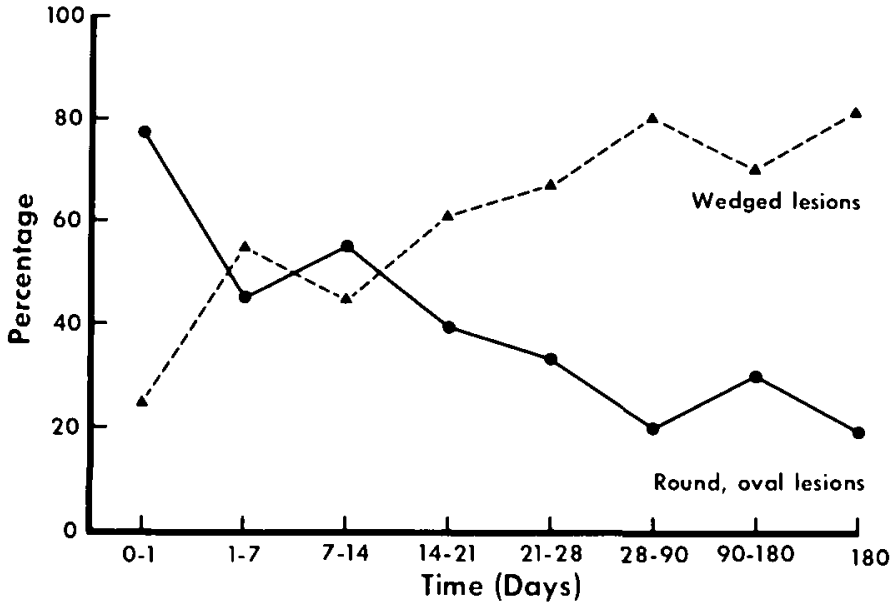

Figure 1 - Percentage of round or oval and wedge-shaped infarcts vs time.

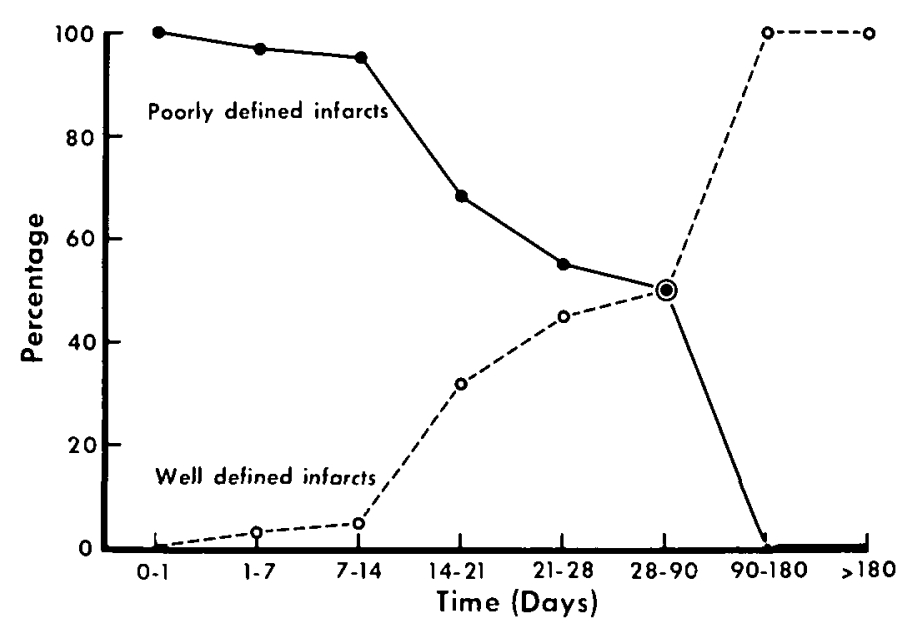

Figure 2 - Percentage of poorly-defined and well-defined infarcts vs time. infarct. Two cases were isodense on the plain study and were delineated only on the infused examination.

The shape of the ischemic infarcts were round, oval or wedged. The classic wedge shape with a central apex and a peripheral base corresponding to the pattern of vascular perfusion became more easily appreciated in the more chronic infarcts. This was likely due to resorption of edema and phagocytosis of necrotic brain. Most infarcts greater than one month of age were wedge-shaped (Fig. I).

Acute infarcts presented with illdefined margins, which over three to four weeks became more clearly demarcated. At three months, most infarcts were well-marginated (Fig. 2).

Mass effect, which ranged from minimal to marked up to the end of the first week, subsided gradually over two or three weeks. After four weeks, the mass effect was usually replaced by loss of volume with ipsilateral ventricular dilatation.

Contrast infusion with subsequent enhancement may alter the appearance of infarcts. The most common alteration was an increase in density. Rarely, there was minimal enhancement of a low density lesion which became isodense and could not be delineated from surrounding brain. This was seen

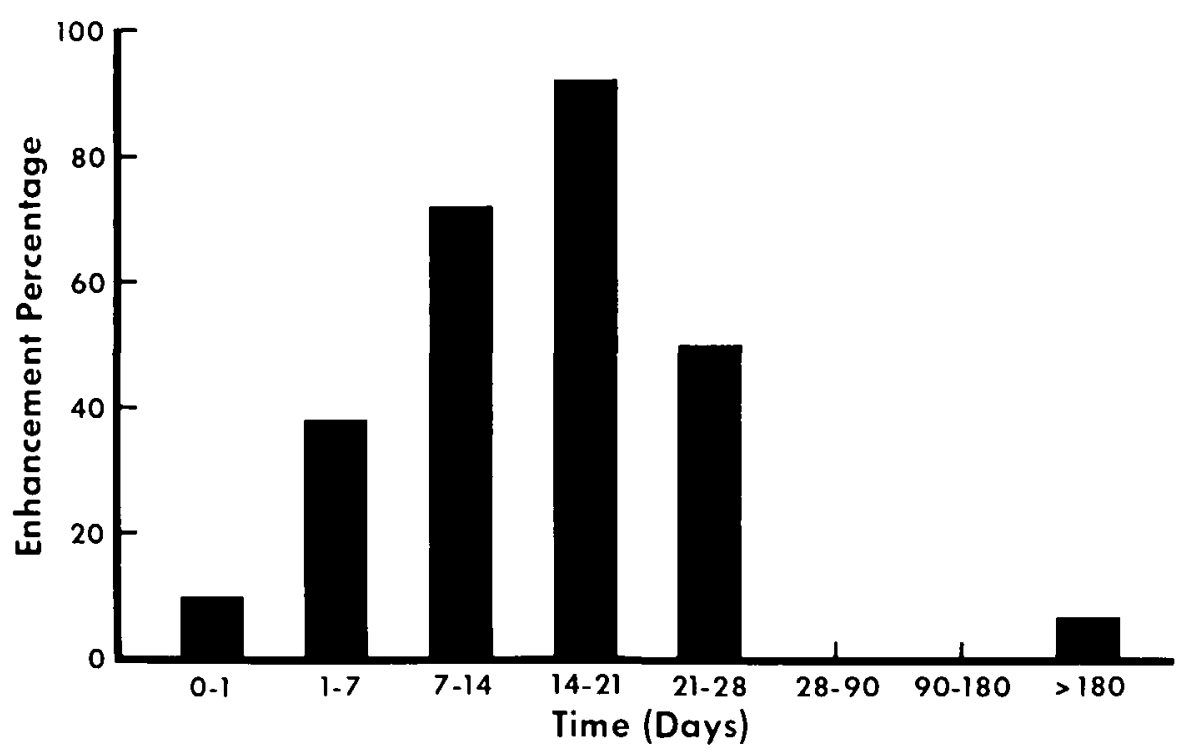

Figure 3 - Percentage of infused scans showing enhancement vs time.

in two cases. Enhancement occurred most commonly one to four weeks following the event although we have observed it from four hours to nine months after the infarction (Fig. 3).

Old infarcts were considered to be those older than four weeks. After this time, infarcts characteristically were wedge-shaped, well-defined, homogeneous areas of low density. Ipsilateral ventricular dilatation was often present ( $54 \%$ of cases).

\section{DISCUSSION}

Cerebral infarctions were usually silent on computed tomography immediately after the ictus. Changes may occur between six and twenty-four hours later (Fig. 4, A \& B) (Yock, 1975).

Most ischemic infarcts presented as an ill-defined zone of decreased density usually extending to the cortex. Edema was probably the most important factor in defining the abnormal zone. The size of the ischemic insult was also important. It was not possible to quantitate the contribution of ischemia and edema in the production of brain lucency with CT. Large cerebral and cerebellar infarcts (Fig. 5) were easily demonstrated. Basal ganglion, thalamus, and internal capsule infarcts were usually well shown. The diminutive infarcts of the brain stem were difficult to assess and were not seen on any of the twenty examinations of clinically diagnosed brain stem disease. Part of the 

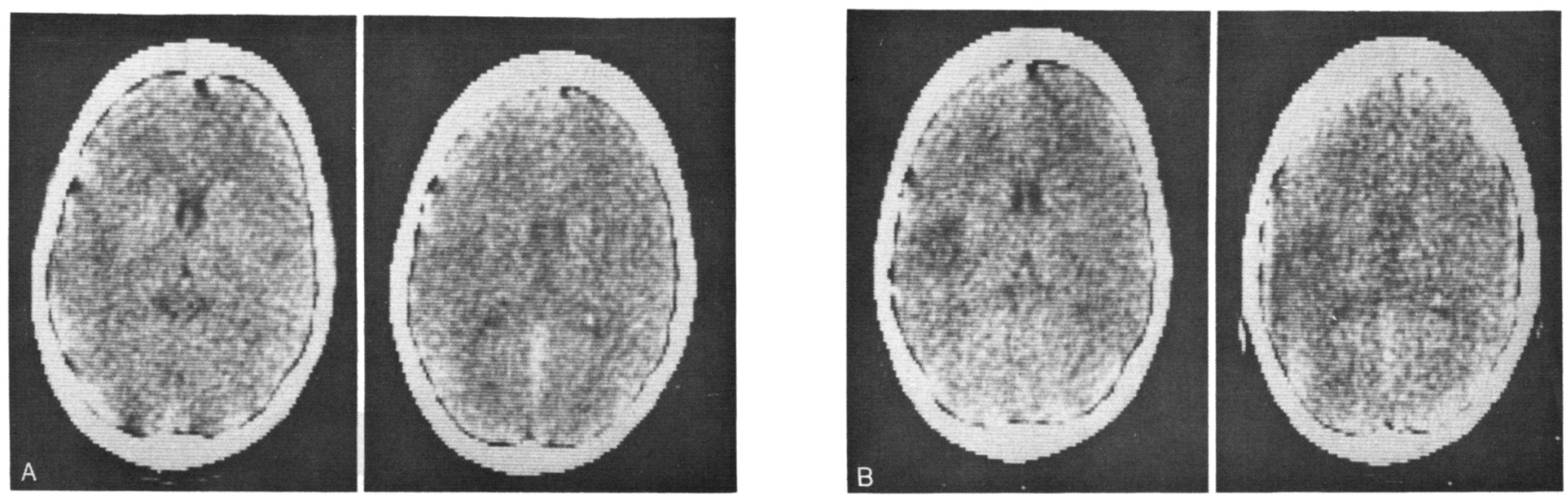

Figure 4 - A) A 27-year old female presented with dysphasia, right hemiparesis, and hypoesthesia 6 hours prior to $\mathrm{Cr}$ scanning. An illdefined minimal decreased density is noted in the left temporo-insular region. B) The left temporo-insular decreased density is now well seen at 24 hours following the ictus.

difficulty in identifying brain stem infarcts was due to artifacts from the petrous tips and clivus which obscured the fine detail in this region.

Involvement of a major vascular territory was an important factor in the diagnosis of an artifact. Lesions of decreased density corresponding to the distribution of the anterior, middle, and posterior cerebral or cerebellar arteries should raise immediate suspicion (Fig. 6). Basal ganglion infarcts can be similarly diagnosed by the presence of four vascular territories corresponding to the anterior, middle, and posterior cerebral; and anterior choroidal artery distribution to the head of the caudate nucleus, internal capsule, thalamus, globus pallidus, and putamen.

Infarcts may be associated with minimal or considerable mass effect. This effect usually subsides in three to four weeks due to edema absorption and phagocytosis of necrotic brain (Lukin, 1977). Contrast enhancement (Ethier, 1974) which is the increase in density seen in abnormal tissue following infusion of iodinated media, aids considerably in the CT assessment of infarction through two possible mechanisms. The most likely etiology of contrast enhancement is due to increased capillary permeability allowing extravasation of contrast medium from the intravascular to the extravascular compartments (Wing, 1978; Gado, 1975). The phenomenon of luxury perfusion, due to alteration of local tissue $\mathrm{pH}$ with loss of vascular autoregulation and arteriovenous shunting may be another cause of contrast enhancement (Lassen, 1964). Thus, both an extravascular and intravascular component may be responsible for enhancement of the brain surrounding infarcted tissue.

Linear, curvilinear, or globular
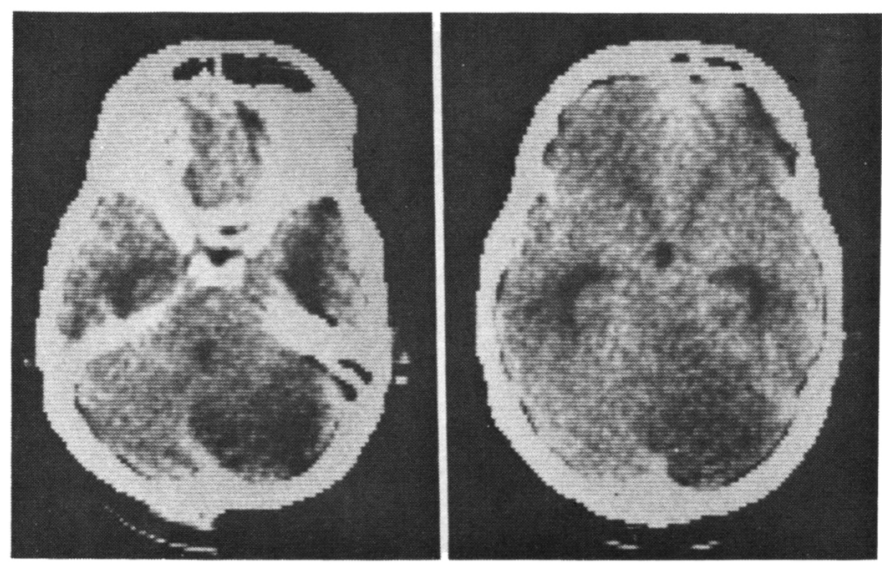

Figure 5 - Obstructive hydrocephalus is present in this 49-year old male with a right cerebellar infarct 24 hours post ictus.
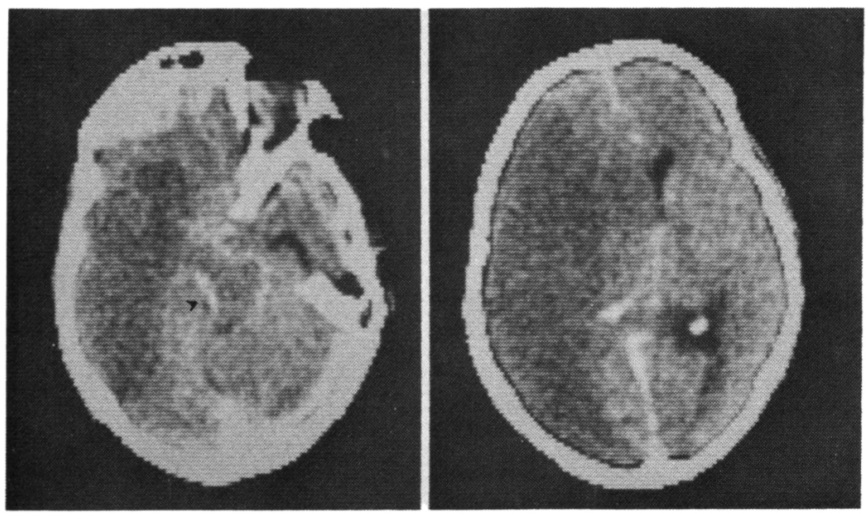

Figure 6 - A large infarct is present involving the entire left middle cerebral territory with sparing of the mesial frontal and occipital lobes. There is a marked mass effect with left posterior hippocampal herniation. which produces medial displacement of the left posterior cerebral artery (proven at autopsy). Black arrow head in the leftsided illustration indicates posterior cerebral artery. 

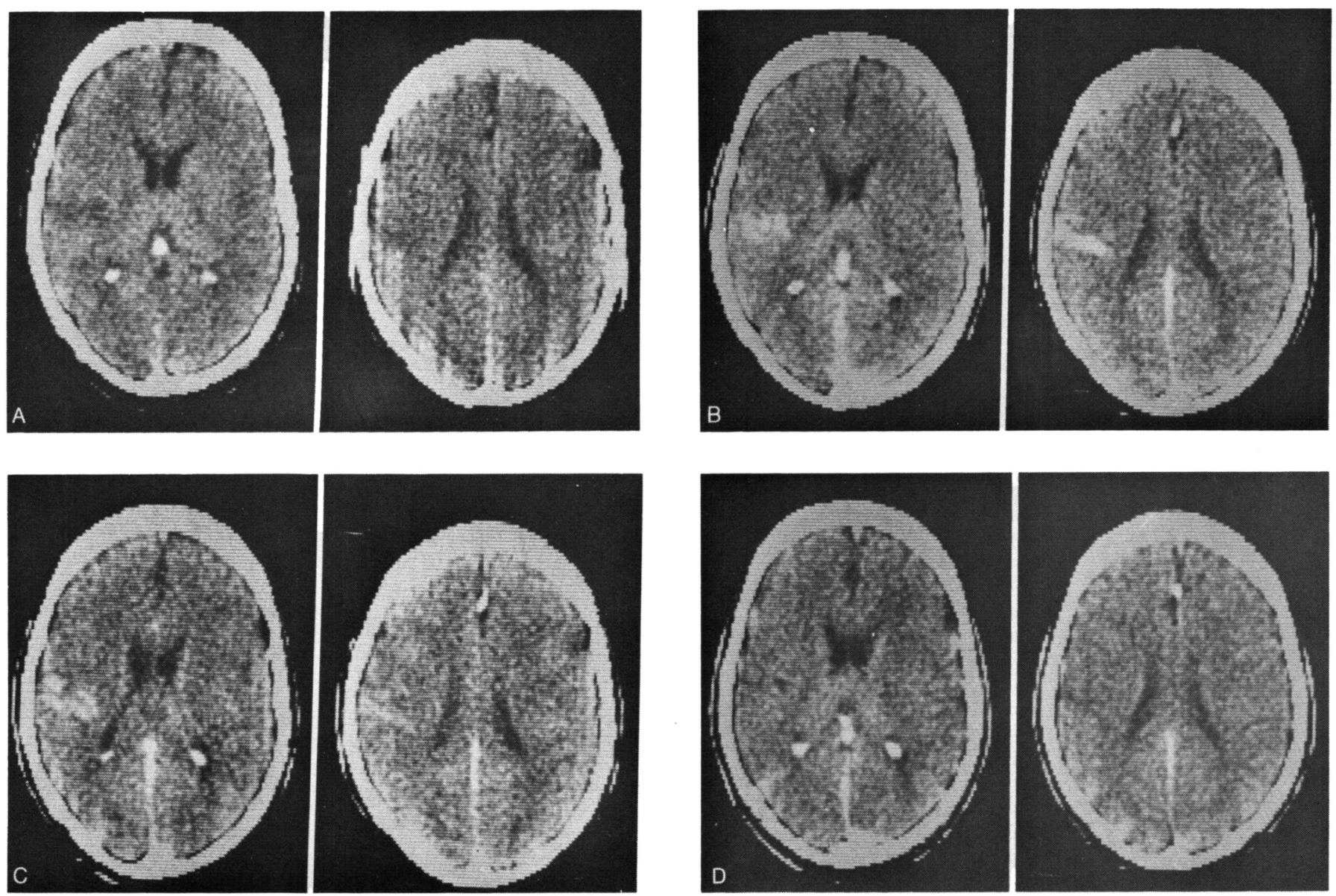

Figure 7 - A) An ill-defined left central infarct one day post ictus. B) Contrast infusion produces almost total wedge-shaped enhancement of the infarct ten days post ictus. C) There is decreasing enhancement eighteen days following the event. D) Twenty-four days post ictus, no enhancement is present; the infarct is smaller and well-defined.

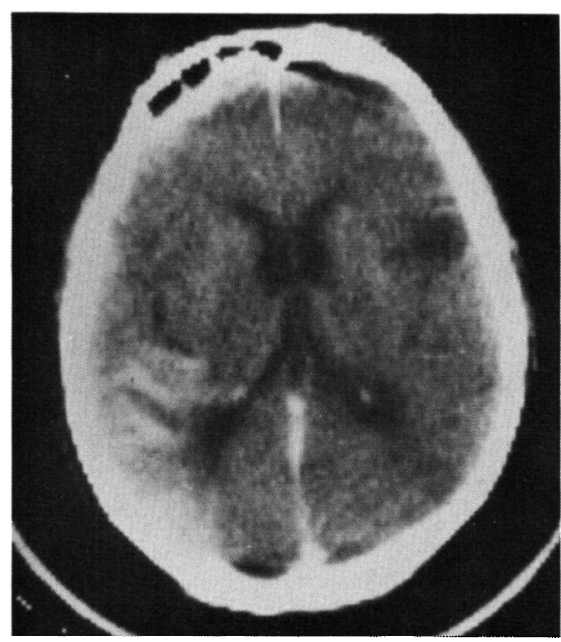

Figure 8 - A recent left posterior tem. poroparietal infarct is present with the enhancement in the posterior aspect assuming the configuration of the cortical gyri. An old right frontotemporal infarct is also present. peripheral increased density, partial or homogeneous, were the most common patterns of contrast enhancement. At times, the entire lesion appeared to enhance (Fig. 7, A, B, C, D) (Wing, 1976). This marked enhancement sometimes assumed the pattern of the cortical gyri (Fig. 8). Complete peripheral or ring opacification similar to that of glioblastoma, abscess or metastases was uncommon (Fig. 9, A, B, C, D) (Lukin, 1977). Contrast enhancement of infarcts permitted visualization of a previously unseen isodense lesion on the plain scan (Fig. 10). It was important to obtain a plain study in order that a hemorrhage into an infarct or hemorrhagic infarct was not confused with marked enhancement following contrast infusion. Rarely, a previously low density lesion was rendered isodense with contrast en- hancement (Fig. 11). It has been stated that false negative results are most likely when scans are limited to only none-contrast or only-contrast techniques (Wing, 1976; Yock, 1976).

Following the initial stages of infarction and edema, there was subsequent necrosis and phagocytosis (Davis, 1975). Phagocytosis usually began at one week and was completed in four to eight weeks. The brain destruction resulted in a variably-sized encephalomalacic cavity and ipsilateral ventricular dilatation (Fig. 12). The cavity may be small and not identifiable, particularly if diffuse cortical atrophy is also present. Hemorrhage into ischemic infarcts is not uncommon (New, 1976). This complication was recognized by the high density of extravasated blood.

During the early experience with 

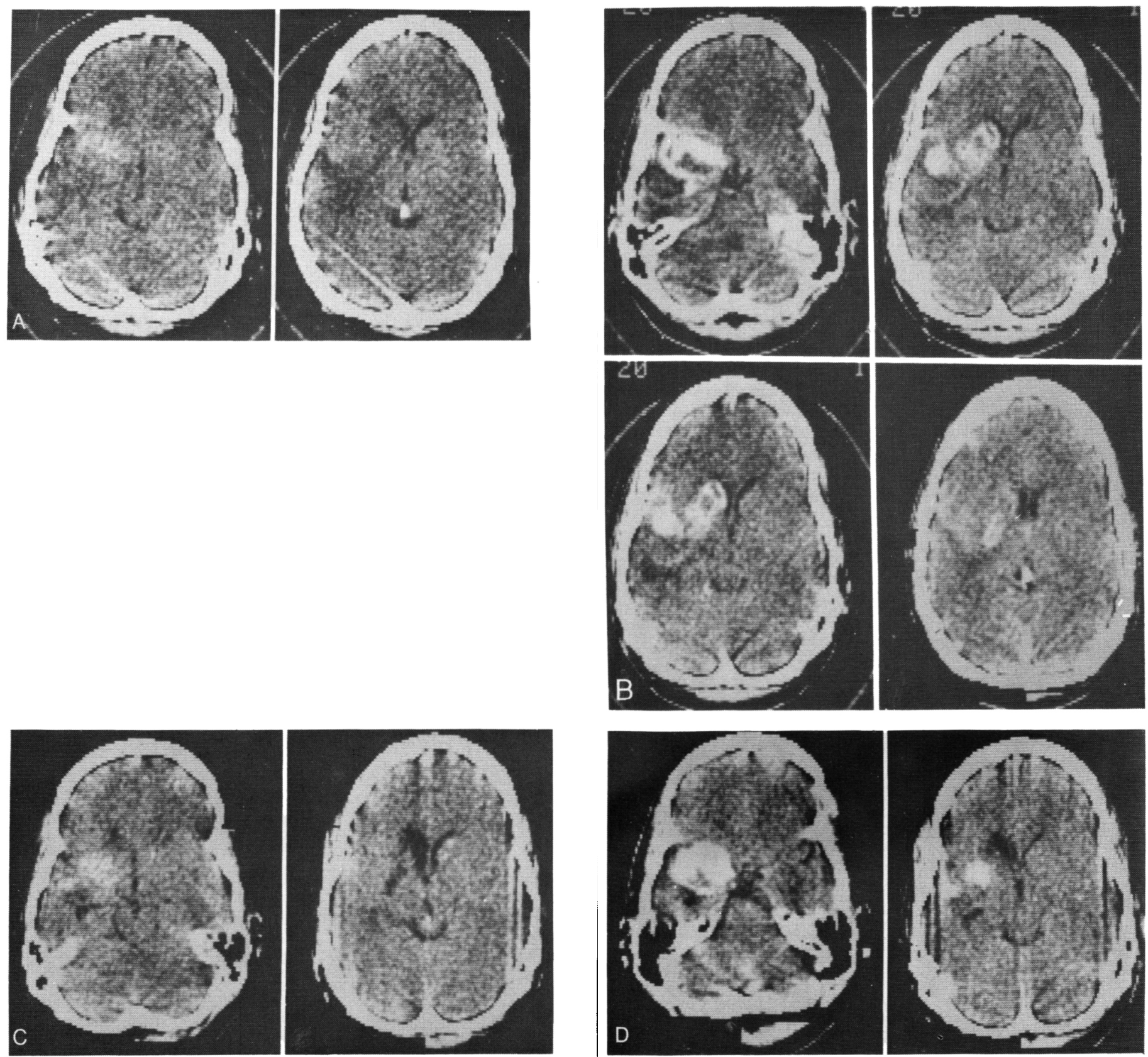

Figure 9 - A) A 21-year old male presented with headache and drowsiness. Six days later, he developed right hemiparesis and receptive aphasia. The initial examinations (not shown) revealed a crescentric left temporal lobe hematoma and giant fusiform aneurysm of the horizontal portion of the left middle cerebral artery. There was no apparent infarct. On the illustrated examination 17 days following onset of hemiparesis and aphasia, ill-defined low density lesions are noted in the head of the left caudate nucleus, anterior limb of the internal capsule and extending into the globus pallidus and insula. B) Infusion at the same time as (A) shows peripherally enhanced infarcts of the left globus pallidus, anterior limb of the internal capsule and head of the caudate nucleus. The aneurysm is partially thrombosed and presents as a fusiform horizontal density. There is enhancement of the capsule of the thrombosed aneurysm wall and left temporal hematoma. C) A follow-up infused examination 2 months following the ictus revealed a well-defined lucency in the left globus pallidus and anterior limb of the internal capsule with localized dilatation of the left frontal horn. D) No enhancement of the infarcts is present on the infused study of that time. The giant left fusiform aneurysm appears larger.

CT, lucencies in the white and grey matter particularly with peripheral contrast enhancement, were thought to represent neoplasia. Angiograms were performed to establish the etiology of the CT abnormality. When the lesion was an infarct the angiogram usually yielded non-specific findings.
Repeat CT scans or surgery were often necessary to be certain of the etiology. Subsequent experience has established a considerable improvement in the 

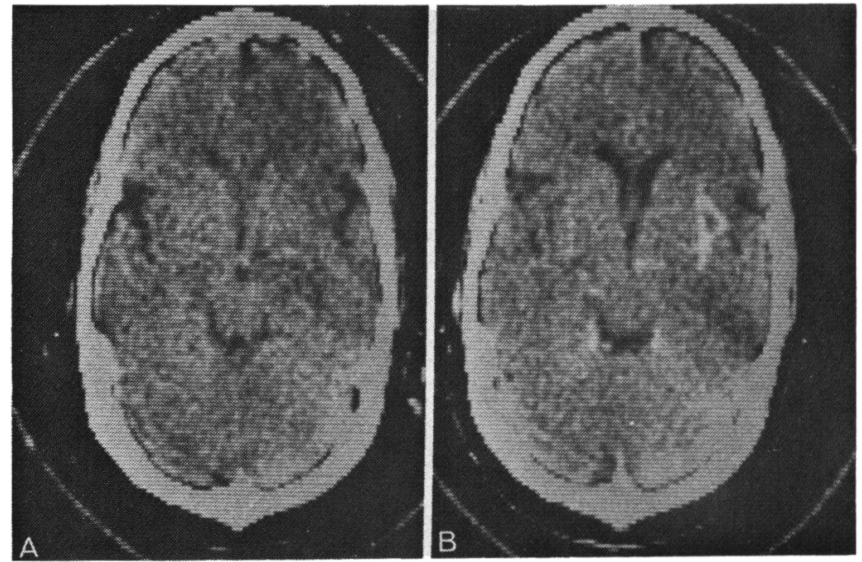

Figure $10-$ A) The non-infused examination 6 weeks post onset of left hemiparesis shows bilateral Sylvian cistern atrophy. B) Contrast infusion reveals right insular enhancement due to infarct.
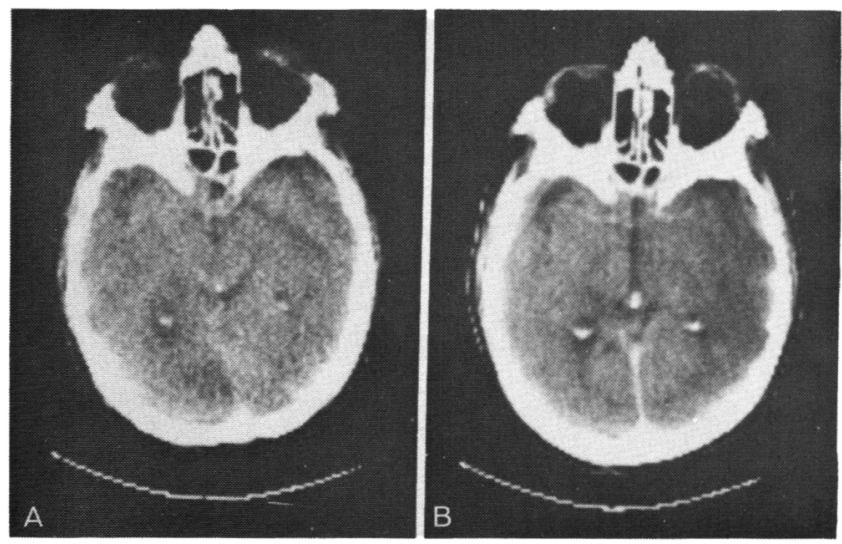

Figure $1 /$ - A) Left occipital decreased density is present due to infarct on the non-infused examination at 3 weeks. B) Infusion of contrast medium at this time produces enough enhancement to obscure the lesion completely.
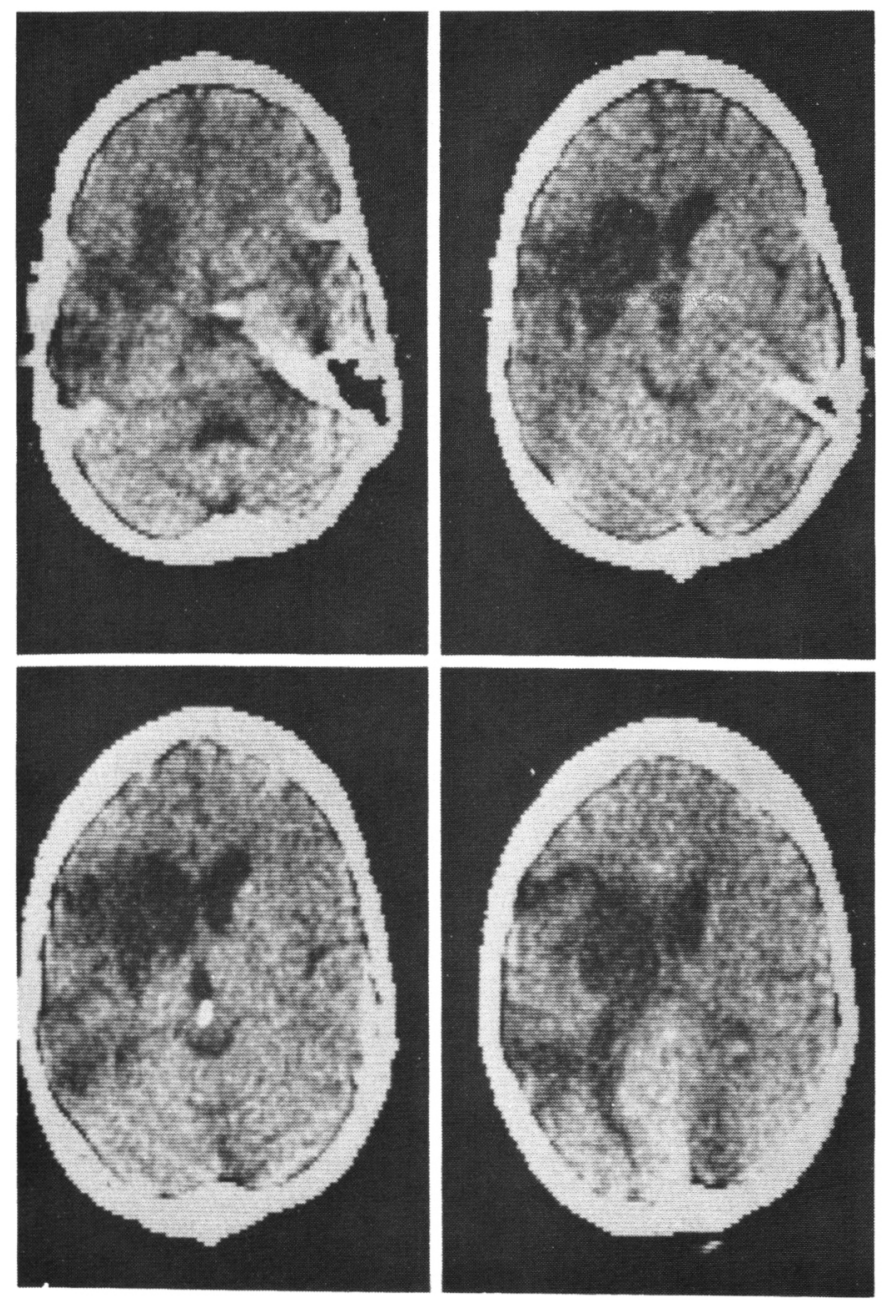

Figure 12 - Eight and one-half months following infarction of the left middle cerebral artery territory with occlusion proximal to the lenticulostriate branches, there is a large well-defined area of encephalomalacia and ipsilateral ventricular dilatation.

accuracy and confidence in differentiating infarcts from tumors.

The CT permits rapid, accurate, and usually specific diagnosis of cerebral infarction. Differentiation from the major diagnostic problem, i.e. tumor, is possible. The method is safe. Angiography need be used only in those patients considered candidates for endarterectomy or extracranialintracranial bypass surgery.

\section{REFERENCES}

CURTIS, B.A. JACOBSON.S. and MARCUS. E.M. (1972). Introduction to the neurosciences. Philadelphia. W.B. Saunders.

DAVIS, K.R., TAVERAS. J.M.. NEW, P.F.J., SCHNUR, J.A., ROBERSON, G.H. (1975). Cerebral infarction diagnosis by computerized tomography. American Journal of Roentgenology 124: 643-660.
ETHIER, R., SHERWIN, A., TAYLOR, S., LEPPIK, I.. THOMPSON, C. (1974). Computerized angio-tomography: the use of $100 \mathrm{cc}$ of Hypaque M-60\%. Clinical and experimental results. Presented at the Symposium on Computerized Tomography, Montreal, Quebec, Canada, May 31 to June 1. 1974.

GADO, M.H., PHELPS, M.E., COLEMAN, R.E. (1975). An extravascular component of contrast enhancement in cranial computed tomography. Radiology 117: 589-597.

LASSEN. N.A. (1964). Autoregulation of cerebral blood flow. Circulation Research 15 (Suppl 1): 1.

LUKIN, R.R., CHAMBERS, A., TOMSICK, T.T. (1977). Cerebral vascular lesions: infarction, hemorrhage, aneurysm, and arteriovenous malformations. Seminars in Roentgenology Vol. XII, No. I.

NEW, P.F., et al (1976). Attenuation measurements of whole blood and blood fractions in computed tomography. Radiology 121: 635640.

TAVERAS, J.M., WOOD, E.H. (1976). Cerebral infarction. (In) Diagnostic Neuroradiology, 2nd Edition, Vol. 2: 888. Baltimore: The Williams and Wilkins Company.

TERBRUGGE, K.. SCOTTI, G.. ETHIER. R.. MELANCCON, D., TCHANG, S., MILNE, C. (1977). Computed tomography in intracranial arteriovenous malformations. Radiology 122: 703-705.

YOCK, D.H. Jr., MARSHALL, W.H. Jr (1975). Recent ischemic brain infarcts at computed tomography. Radiology 117: 599 . 608.

YOCK, D.H., NORMAN, D., POLLOCK, J.A., NEWTON, T.H. (1976). Pitfalls in the computed tomography diagnosis of ischemic infarcts. Presented at the International Symposium on Computed Assisted Tomography in Nontumoural Diseases of the Brain, Spinal Cord and Eye, Bethesda, Maryland. 